\title{
Bericht des AfnP-Vorstands Geschäftsordnung ergänzt Satzung
}

Die Satzung der AfnP wird durch eine Geschäftsordnung um einige Punkte ergänzt. Dies betrifft unter anderem neue Mitgliedschaften und die regionale Gliederung der AfnP. Die finanzielle Lage hat sich gut entwickelt und wir arbeiten konstruktiv mit ärztlichen und pflegerischen Fachverbänden zusammen. Die ursprünglich angedachte Fusion mit dem fnb ist bis auf Weiteres verschoben.

Zusammen mit der Landesvertreterversammlung erarbeiten wir für die AfnP eine Geschäftsordnung, die unsere Satzung um fehlende Punkte ergänzen wird. Folgende Punkte sind in Arbeit:

\section{Mitgliedschaft in der AfnP}

Neuen Mitgliedern, die bis zum 31. Juli ihre Mitgliedschaft beantragen, stehen für das laufende Kalenderjahr alle Leistungen der AfnP zu. Sie erhalten rückwirkend die Ausgaben der Zeitschrift Dialyse aktuell des ersten Halbjahres und bekommen freien Eintritt beim jährlichen Symposium. Mitgliedern, die ab August in die AfnP eintreten, können wir diese Vergünstigungen nicht gewähren. Sie erhalten eine farbig gesonderte Mitgliedskarte. Kündigungen müssen vor dem Ablauf des Kalenderjahres, bis zum 30. September schriftlich bei der Geschäftsstelle eingegangen sein, sonst verlängert sich die Mitgliedschaft automatisch um ein weiteres Kalenderjahr.
Wir möchten die selbstzahlenden Mitglieder bitten, bis spätestens 31. Januar ihren Mitgliedsbeitrag auf das Konto der AfnP zu überweisen

Sparkasse Ulm

BLZ: 63050000

IBAN: DE 85630500000021095551

Kontonummer: 21095551

Im Februar erhalten alle Mitglieder, die Ihren Mitgliedsbeitrag bezahlt haben, ihren Mitgliedsausweis. Bitte melden Sie rechzeitig Änderungen ihrer Mitgliedsdaten oder ihrer Bankverbindung schriftlich an die Geschäftsstelle, um unnötige Kosten zu vermeiden. Indem Sie der AfnP eine Einzugsermächtigung erteilen, erleichtern Sie uns die Arbeit und helfen, Portokosten einzusparen.

\section{Aufgaben und Struktur der AfnP}

Die Afnp ist in drei Organe gegliedert

- Vorstand, der von den Mitgliedern gewählt wird

- Ländervertreterversammlung

- Mitgliederversammlung.

Das genaue Ergebnis der Geschäftsordnung bezüglich der regionalen Gliederung der AfnP und der Aufgabenverteilung stellen wir Ihnen auf der Mitgliederversammlung am Samstag, 25.10.2008 um 17.00 Uhr in Fulda vor.

\section{Die Situation der AfnP}

Unsere Kooperation mit den ärztlichen und pflegerischen Fachverbänden läuft für alle Beteiligten sehr konstruktiv. In letzter Zeit ist es immer wieder zur Ent-

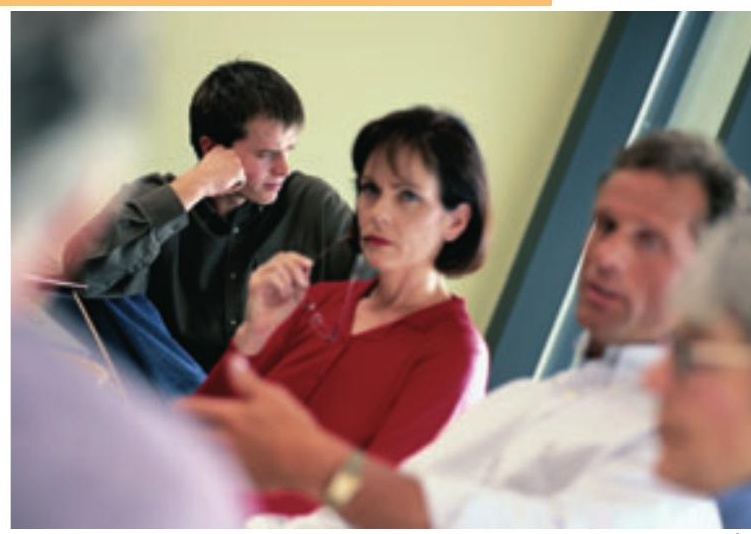

stehung von Unklarheiten und Gerüchten gekommen, die wir gerne klarstellen möchten. Die AfnP wird sich nicht auflösen. Gerade haben wir einen Fünfjahresvertrag mit dem Maritim Hotel in Fulda geschlossen. Ebenso wie in diesem Jahr findet auch in den darauffolgenden vier Jahren wieder Ende Oktober das AfnPSymposium in Fulda statt.

Die finanzielle Lage der AfnP hat sich durch das umsichtige Agieren im Sommer 2007 sehr gut entwickelt. Wir können nun auf ein Polster von Rücklagen zurückgreifen und dies mitgliederorientiert einsetzen. Die Bilanzen 2006 und 2007, die beim Symposium noch nicht vorlagen, sind den Mitgliedern mit ihren Mitgliedskarten zugestellt worden. Die Kassenprüfung für das Jahr 2007 ist Ende Februar ohne Beanstandung erfolgt. Zurzeit bauen wir mithilfe unserer teilweise neuen, sehr aktiven Ländervertreter die regionalen Fortbildungen (FB) aus und hoffen, bald wieder mehr Seminare in Fulda anbieten zu können.

\section{Beseitigung von Unklarheiten}

Bezüglich der Zusammenarbeit mit dem Fachverband für nephrologische Berufsgruppen (fnb) möchten wir an dieser Stelle zur Aufklärung und Beseitigung von Unklarheiten beitragen. Fälschlicherweise wird oft angenommen, der fnb ist der Dachverband der nephrologischen Pflege in Deutschland. Dies ist nicht der Fall. Wie auch die AfnP ist der fnb ein eigenständiger Berufsverband für die nephrologische Pflege, der von den ehemaligen Mitgliedern des deutschen Zweiges der EDTNA/ERCA am 01. Januar 2006 gegründet worden ist. Den zuvor bestehenden langjährigen Kooperationsvertrag mit der AfnP hat der Vorstand des deutschen Zweiges (Christa Tast) im Vorfeld gekündigt.

Ebenfalls im Vorfeld, im Frühjahr 2005, haben die Vertreter des deutschen Zweiges der EDTNA/ERCA gemeinsam mit den Vertretern des AfnP alle Vor- 
und Nachteile eines gemeinsamen nationalen nephrologischen Berufsverbands diskutiert, konnten jedoch keinen Konsens finden.

Daher hat sich im August 2005 der deutsche Zweig entschlossen, einen neuen, eigenständigen Berufsverband zu gründen. Ein Motiv zur Neugründung des fnb war damals der Wunsch, kein Anhängsel der großen internationalen EDTNA/ ERCA zu sein, sondern ein „eigenständiger Verband“. Mit einer eigenen Satzung und einem eigenen Logo sind sie nun unabhängig von der EDTNA - auch was die Verwaltung der generierten Mitgliedsbeiträge betrifft. Ein weiterer Ablösungsgrund war die angestrebte Fusion mit der AfnP (Forum 01/2006). Mit einem eigenen, mitgliederstarken Verband sollte der fnb als gleichberechtigter Partner gegenüber der AfnP auftreten können.

\section{Gemeinsame Projekte ja, Fusion nein}

Die weitere Zusammenarbeit gestaltete sich in der Folgezeit nicht ganz einfach. Die Kommunikation in gemeinsamen Arbeitsgruppen lief nicht rund und immer wieder traten Missverständnisse auf. Anfang 2007 haben Vorstandsmitglieder des fnb erklärt, dass der fnb entgegen seiner Verlautbarung im Forum 02/2007 an keiner Zusammenarbeit/Kooperation interessiert ist. Dies müssen wir akzeptieren.

In einer erweiterten Vorstandssitzung Ende Februar haben wir diese Punkte noch einmal lange erörtert und sind zu folgendem Beschluss gekommen: Gespräche bezüglich einer Fusion von AfnP und fnb werden aufgrund der schwierigen Kommunikation über die Ziele der jeweiligen Verbände auf unbestimmte Zeit ausgesetzt, was aber eine Zusammenarbeit beider Verbände nicht ausschließt. AfnP und fnb werden also weiterhin gemeinsam einzelne Projekte planen und in paritätischen Arbeitsgruppen zusammenarbeiten. Vereinbarungen hierüber werden künftig schriftlich festgelegt.

\section{Situation der nephrologischen Pflege in Deutschland}

So haben wir in Deutschland für die nephrologische Pflege folgende Situation: Es gibt im Bereich nephrologische Pflege folgende Verbände

- AfnP e. V

- AKTX-Pflege e. V.

- fnb e. V

- EDTNA/ERCA deutscher Zweig.

Die AfnP hat Kooperationsverträge mit dem AKTX-Pflege und der EDTNA/ERCA International. Die Gesellschaft für Nephrologie (GfN), die Psychonephrologen und der AKTX unterstützen uns bei der jährlichen Programmgestaltung für das Symposium.

Wir werden auch weiterhin das offene und ehrliche Gespräch mit allen Berufsgruppen suchen und ausbauen. Mit einem Netzwerk der nephrologischen Interessensverbände können wir maßgeblich Einfluss auf Veränderungen und Entwicklungen im Gesundheitswesen nehmen. Um im Pflegebereich etwas zu erreichen, ist es notwendig, gemeinsam mit einer Stimme aufzutreten und zu handeln. Diese Kooperationen hat die AfnP in den letzten Jahren ständig ausgebaut und dazu viele sachorientierte, konstruktive Gespräche geführt und wird dies auch weiterhin tun. 